\title{
ON ANISOTROPIC ELASTIC MATERIALS THAT POSSESS THREE IDENTICAL STROH EIGENVALUES AS DO ISOTROPIC MATERIALS
}

By

\author{
T. C. T. TING
}

University of Illinois at Chicago

\begin{abstract}
For anisotropic elastic materials for which the displacements $u_{i}$ depend on $x_{1}$ and $x_{2}$ only, a general solution for $u_{i}$ depends on one variable $z=x_{1}+p x_{2}$ where $p$ is an eigenvalue of the fundamental elasticity tensor of Stroh. There are six $p$ 's which consist of three pairs of complex conjugates. For isotropic materials, $p= \pm i$ are the eigenvalues of multiplicity three. We point out trivial cases in which a completely anisotropic material has the eigenvalues $p= \pm i$ and has the solutions to two-dimensional elasticity problems that are identical to the solutions for isotropic materials. Excluding these trivial cases, we show that $p= \pm i$ can be the eigenvalues of multiplicity three for monoclinic materials with the symmetry plane at $x_{1}=0$, at $x_{2}=0$, or at any plane that contains the $x_{3}$-axis. If the symmetry plane is at $x_{3}=0$, then $p= \pm i$ occur only when the material is transversely isotropic with the axis of symmetry at the $x_{3}$-axis. We also consider the general case in which the eigenvalues are arbitrary and are of multiplicity three. The eigenrelation associated with the triple eigenvalues is nonsemisimple for all cases studied here. There are only two independent eigenvectors associated with the triple eigenvalues.
\end{abstract}

1. Introduction. In a fixed rectangular coordinate system $x_{i}(i=1,2,3)$ let $u_{i}, \sigma_{i j}$, respectively, be the displacement and stress in an anisotropic elastic material. The stress-strain laws and the equations of equilibrium are

$$
\begin{aligned}
& \sigma_{i j}=C_{i j k s} u_{k, s}, \\
& C_{i j k s} u_{k, s j}=0,
\end{aligned}
$$

where a comma stands for differentiation, repeated indices imply summation, and the $C_{i j k s}$ are the elastic stiffnesses which are assumed to be fully symmetric and positive definite. For two-dimensional deformations in which $u_{i}$ depends on $x_{1}, x_{2}$ only, a general solution to Eq. (1.2) is, in matrix notation,

$$
\mathbf{u}=\mathbf{a} f(z), \quad z=x_{1}+p x_{2} .
$$

Received May 3, 1992. 
In the above $f$ is an arbitrary function of $z$, and $p$ and a are determined by inserting Eq. (1.3) into Eq. (1.2). We have

$$
\left\{\mathbf{Q}+p\left(\mathbf{R}+\mathbf{R}^{\mathrm{T}}\right)+p^{2} \mathbf{T}\right\} \mathbf{a}=0
$$

where the superscript $\mathrm{T}$ denotes the transpose and $\mathbf{Q}, \mathbf{R}, \mathbf{T}$ are $3 \times 3$ real matrices whose components are

$$
Q_{i k}=C_{i 1 k 1}, \quad R_{i k}=C_{i 1 k 2}, \quad \mathrm{~T}_{i k}=C_{i 2 k 2} .
$$

It is seen that $\mathbf{Q}$ and $\mathbf{T}$ are symmetric and positive definite. For a nontrivial solution of a from Eq. (1.4) we must have

$$
\left|\mathbf{Q}+p\left(\mathbf{R}+\mathbf{R}^{\mathrm{T}}\right)+p^{2} \mathbf{T}\right|=0 .
$$

There are six eigenvalues $p$ from Eq. (1.6) which, if the strain energy is positive, consist of three pairs of complex conjugates $[1,2]$. If $p_{\alpha}, \alpha=1,2, \ldots, 6$, are the eigenvalues, we let

$$
\operatorname{Im} p_{\alpha}>0, \quad \alpha=1,2,3,
$$

where Im stands for the imaginary part. For isotropic materials it is known that

$$
p_{1}=p_{2}=p_{3}=i=\sqrt{-1} \text {. }
$$

The purpose of this paper is to show that there are infinitely many anisotropic elastic materials whose eigenvalues are given by Eq. (1.7). In fact, we will also show that there are infinitely many anisotropic materials for which the three identical eigenvalues can be arbitrary.

2. Dependence of $p$ on elasticity constants. Employing the contracted notation $C_{p q}$ for $C_{i j k s}$, the three matrices $\mathbf{Q}, \mathbf{R}$, and $\mathbf{T}$ of Eqs. (1.5) have the expressions

$$
\begin{aligned}
\mathbf{Q} & =\left[\begin{array}{lll}
C_{11} & C_{16} & C_{15} \\
C_{61} & C_{66} & C_{65} \\
C_{51} & C_{56} & C_{55}
\end{array}\right], \\
\mathbf{R} & =\left[\begin{array}{lll}
C_{16} & C_{12} & C_{14} \\
C_{66} & C_{62} & C_{64} \\
C_{56} & C_{52} & C_{54}
\end{array}\right], \\
\mathbf{T} & =\left[\begin{array}{lll}
C_{66} & C_{62} & C_{64} \\
C_{26} & C_{22} & C_{24} \\
C_{46} & C_{42} & C_{44}
\end{array}\right],
\end{aligned}
$$

and Eq. (1.6) leads to a sextic equation for $p$. An alternative sextic equation for $p$ is [3]

$$
l_{2}(p) l_{4}(p)-l_{3}(p) l_{3}(p)=0
$$

where

$$
\begin{aligned}
& l_{2}(p)=s_{55}^{\prime} p^{2}-2 s_{45}^{\prime} p+s_{44}^{\prime}, \\
& l_{3}(p)=s_{15}^{\prime} p^{3}-\left(s_{14}^{\prime}+s_{56}^{\prime}\right) p^{2}+\left(s_{25}^{\prime}+s_{46}^{\prime}\right) p-s_{24}^{\prime}, \\
& l_{4}(p)=s_{11}^{\prime} p^{4}-2 s_{16}^{\prime} p^{3}+\left(2 s_{12}^{\prime}+s_{66}^{\prime}\right) p^{2}-2 s_{26}^{\prime} p+s_{22}^{\prime} .
\end{aligned}
$$


In the above, $s_{i j}^{\prime}$ are the reduced elastic compliances which are related to the elastic compliances $s_{i j}$ by

$$
s_{i j}^{\prime}=s_{i j}-\frac{s_{i 3} s_{3 j}}{s_{33}}
$$

Written as a $6 \times 6$ matrix, $s_{i j}$ is the inverse of $C_{i j}$. Both $s_{i j}$ and $C_{i j}$ are symmetric and positive definite. The $s_{i j}^{\prime}$ defined in Eq. (2.3) vanish for $i=3$ and/or $j=$ 3. Removing the third row and the third column of $s_{i j}^{\prime}$ which contain only zero elements, we have a $5 \times 5$ symmetric matrix $s_{i j}^{\prime}$ given by

$$
\mathbf{s}^{\prime}=\left[\begin{array}{lllll}
s_{11}^{\prime} & s_{12}^{\prime} & s_{14}^{\prime} & s_{15}^{\prime} & s_{16}^{\prime} \\
s_{21}^{\prime} & s_{22}^{\prime} & s_{24}^{\prime} & s_{25}^{\prime} & s_{26}^{\prime} \\
s_{41}^{\prime} & s_{42}^{\prime} & s_{44}^{\prime} & s_{45}^{\prime} & s_{46}^{\prime} \\
s_{51}^{\prime} & s_{52}^{\prime} & s_{54}^{\prime} & s_{55}^{\prime} & s_{56}^{\prime} \\
s_{61}^{\prime} & s_{62}^{\prime} & s_{64}^{\prime} & s_{65}^{\prime} & s_{66}^{\prime}
\end{array}\right]
$$

It can be shown [4] that the following $5 \times 5$ symmetric matrix,

$$
\mathbf{C}^{0}=\left[\begin{array}{lllll}
C_{11} & C_{12} & C_{14} & C_{15} & C_{16} \\
C_{21} & C_{22} & C_{24} & C_{25} & C_{26} \\
C_{41} & C_{42} & C_{44} & C_{45} & C_{46} \\
C_{51} & C_{52} & C_{54} & C_{55} & C_{56} \\
C_{61} & C_{62} & C_{64} & C_{65} & C_{66}
\end{array}\right],
$$

which is obtained from $C_{i j}$ by deleting the third row and the third column of $C_{i j}$, is the inverse of $s_{i j}^{\prime}$. Hence

$$
\mathbf{s}^{\prime} \mathbf{C}^{0}=\mathbf{I},
$$

where $\mathbf{I}$ is the $5 \times 5$ unit matrix. Since $\mathbf{C}^{0}$ is positive definite, so is $\mathbf{s}^{\prime}$.

Equation (1.6) depends on the 15 constants of $\mathbf{C}^{0}$ only. The constants $C_{i 3}$, $i=1,2, \ldots, 6$, do not appear in Eq. (1.6). Therefore, one can have an anisotropic material whose $C_{i j}$ are identical to those of isotropic materials except $C_{i 3}$, i.e.,

$$
C_{i j}=\left[\begin{array}{cccccc}
\lambda+2 \mu & \lambda & C_{13} & 0 & 0 & 0 \\
\lambda & \lambda+2 \mu & C_{23} & 0 & 0 & 0 \\
C_{13} & C_{23} & C_{33} & C_{43} & C_{53} & C_{63} \\
0 & 0 & C_{43} & \mu & 0 & 0 \\
0 & 0 & C_{53} & 0 & \mu & 0 \\
0 & 0 & C_{63} & 0 & 0 & \mu
\end{array}\right],
$$

where $\lambda$ and $\mu$ are the Lamé constants. The eigenvalues of Eq. (2.7) are given by Eq. (1.7). Likewise, since Eq. (2.1) depends on the 15 constants of $\mathbf{s}^{\prime}$, we can have the following anisotropic materials whose $\mathbf{s}^{\prime}$ is identical to the $\mathbf{s}^{\prime}$ of isotropic materials but whose $s_{i j}$ obtained from Eq. (2.3) are different by choosing $s_{i 3}$ arbitrarily. We 
have

$$
s_{i j}=\frac{1}{E}\left[\begin{array}{cccccc}
1-\nu^{2}+\varepsilon_{1}^{2} & -\nu(1+\nu)+\varepsilon_{1} \varepsilon_{2} & \varepsilon_{1} \varepsilon_{3} & \varepsilon_{1} \varepsilon_{4} & \varepsilon_{1} \varepsilon_{5} & \varepsilon_{1} \varepsilon_{6} \\
-\nu(1+\nu)+\varepsilon_{1} \varepsilon_{2} & 1-\nu^{2}+\varepsilon_{2}^{2} & \varepsilon_{2} \varepsilon_{3} & \varepsilon_{2} \varepsilon_{4} & \varepsilon_{2} \varepsilon_{5} & \varepsilon_{2} \varepsilon_{6} \\
\varepsilon_{1} \varepsilon_{3} & \varepsilon_{2} \varepsilon_{3} & \varepsilon_{3}^{2} & \varepsilon_{3} \varepsilon_{4} & \varepsilon_{3} \varepsilon_{5} & \varepsilon_{3} \varepsilon_{6} \\
\varepsilon_{1} \varepsilon_{4} & \varepsilon_{2} \varepsilon_{4} & \varepsilon_{3} \varepsilon_{4} & 2(1+\nu)+\varepsilon_{4}^{2} & \varepsilon_{4} \varepsilon_{5} & \varepsilon_{4} \varepsilon_{6} \\
\varepsilon_{1} \varepsilon_{5} & \varepsilon_{2} \varepsilon_{5} & \varepsilon_{3} \varepsilon_{5} & \varepsilon_{4} \varepsilon_{5} & 2(1+\nu)+\varepsilon_{5}^{2} & \varepsilon_{5} \varepsilon_{6} \\
\varepsilon_{1} \varepsilon_{6} & \varepsilon_{2} \varepsilon_{6} & \varepsilon_{3} \varepsilon_{6} & \varepsilon_{4} \varepsilon_{6} & \varepsilon_{5} \varepsilon_{6} & 2(1+\nu)+\varepsilon_{6}^{2}
\end{array}\right]
$$

where $\varepsilon_{i}, i=1,2, \ldots, 6$, are arbitrary constants and

$$
E=\frac{\mu(3 \lambda+2 \mu)}{\lambda+\mu}, \quad \nu=\frac{\lambda}{2(\lambda+\mu)}
$$

are Young's modulus and Poisson's ratio, respectively. The eigenvalues of Eq. (2.8) are given by Eq. (1.7). In fact, the solutions to two-dimensional elastostatics problems and steady-state motions such as surface waves for the materials given by Eq. (2.7) or Eq. (2.8) are identical to the solutions for isotropic materials. The $s_{i j}$ of Eq. (2.8) represent isotropic materials when $\varepsilon_{1}=\varepsilon_{2}=-\nu, \varepsilon_{3}=1$, and $\varepsilon_{4}=\varepsilon_{5}=\varepsilon_{6}=0$. If $\varepsilon_{i}$ (and $C_{i 3}$ ) are chosen arbitrarily, the materials represented by Eq. (2.7) or Eq. (2.8) can be completely anisotropic without a plane of material symmetry [5]. The matrix $s_{i j}$ of Eq. (2.8) is positive definite if

$$
E>0, \quad-1<\nu<\frac{1}{2}, \quad \varepsilon_{3} \neq 0 .
$$

We will verify Eq. (2.9) shortly. Since the matrices $C_{i j}$ of Eq. (2.7) and $s_{i j}$ of Eq. (2.8) are the inverses of each other, we obtain $C_{i 3}$ in terms of $\varepsilon_{i}$ (and hence of $s_{i 3}$ ) as

$$
\begin{aligned}
& C_{13}=-\frac{1}{s_{33}}\left[\lambda\left(s_{13}+s_{23}\right)+2 \mu s_{13}\right], \\
& C_{23}=-\frac{1}{s_{33}}\left[\lambda\left(s_{13}+s_{23}\right)+2 \mu s_{23}\right], \\
& C_{33}=\frac{1}{s_{33}}+\left[\frac{1}{s_{33}}\right]^{2}\left\{\lambda\left(s_{13}+s_{23}\right)^{2}+\mu\left(2 s_{13}^{2}+2 s_{23}^{2}+s_{43}^{2}+s_{53}^{2}+s_{63}^{2}\right)\right\}, \\
& C_{i 3}=-\mu \frac{s_{i 3}}{s_{33}}, \quad i=4,5,6 .
\end{aligned}
$$

With the $C_{i 3}$ given above, the matrix $C_{i j}$ of Eq. (2.7) is positive definite when $s_{i j}$ is. Anisotropic materials such as those given by Eq. (2.7) or Eq. (2.8) for which $\mathbf{s}^{\prime}$ and $\mathbf{C}^{0}$ are identical to those of isotropic materials will be excluded in the sequel.

In the rest of the paper we consider $\mathbf{C}^{0}$ and $\mathbf{s}^{\prime}$ which are different from those for isotropic materials. When $\mathbf{s}^{\prime}$ is positive definite, it is very simple to choose $s_{i 3}, i=1,2, \ldots, 6$, such that $s_{i j}$ is positive definite. All we have to do is choose $s_{33}>0$. To see this, let $\xi_{i}, i=1,2, \ldots, 6$, be arbitrary real constants, not all of them zero. We have from Eq. (2.3),

$$
\sum_{i=1}^{6} \sum_{j=1}^{6} s_{i j} \xi_{i} \xi_{j}=\sum_{i=1}^{6} \sum_{j=1}^{6} s_{i j}^{\prime} \xi_{i} \xi_{j}+\frac{1}{s_{33}}\left\{\sum_{i=1}^{6} s_{i 3} \xi_{i}\right\}^{2} .
$$


With $s_{33}>0$, the right-hand side is always positive. It vanishes only when all $\xi_{i}$ vanish, confirming that $s_{i j}$ is positive definite. The matrix $s_{i j}$ of Eq. (2.8) subject to Eqs. (2.9) is positive definite because $s_{33}>0$, and the first two conditions of Eqs. (2.9) assure us that the associated $\mathbf{s}^{\prime}$ derived from Eq. (2.8) is positive definite.

If $\mathbf{C}^{0}$ is positive definite, it is always possible to choose $C_{i 3}$ (without first determining $s_{i 3}$ ), such that $\mathbf{C}$ is positive definite. To do this, we observe that

$$
|\mathbf{C}|=C_{33}\left|\mathbf{C}^{0}\right|-\mathbf{h}^{\mathrm{T}} \mathbf{C}^{0^{*}} \mathbf{h}
$$

where

$$
\mathbf{h}^{\mathrm{T}}=\left(C_{13}, C_{23}, C_{43}, C_{53}, C_{63}\right)
$$

and $\mathbf{C}^{0^{*}}$ is the adjoint of $\mathbf{C}^{0}$ so that

$$
\mathbf{C}^{0} \mathbf{C}^{0^{*}}=\left|\mathbf{C}^{0}\right| \mathbf{I} \text {. }
$$

Hence Eq. (2.11) can be written as

$$
\frac{|\mathbf{C}|}{\left|\mathbf{C}^{0}\right|}=C_{33}-\mathbf{h}^{\mathrm{T}}\left(\mathbf{C}^{0}\right)^{-1} \mathbf{h} \text {. }
$$

If $\mathbf{C}^{0}$ is positive definite, so is $\mathbf{C}$ provided $|\mathbf{C}|>0$ [6]. Therefore, if we choose

$$
C_{33}>\mathbf{h}^{\mathrm{T}}\left(\mathbf{C}^{0}\right)^{-1} \mathbf{h}=\mathbf{h}^{\mathrm{T}} \mathbf{s}^{\prime} \mathbf{h}
$$

where the equality follows from Eq. (2.6), we have a positive definite $\mathbf{C}$.

The sextic equation (2.1) has one advantage in that each component of $\mathrm{s}^{\prime}$ appears only once in the equation. For the sextic equation (1.6), some components of $\mathbf{C}^{0}$ appear more than once. We will therefore begin with Eq. (2.1) and, after determining a positive definite $\mathbf{s}^{\prime}$ for which the eigenvalues are given by Eq. (1.7), obtain a complete set of $s_{i j}$ from Eq. (2.3) by choosing $s_{i 3}$ arbitrarily with $s_{33}>0$. As to a complete set of positive definite $C_{i j}$, we use Eq. (2.6) to obtain $C_{i j}^{0}$ and choose $C_{i 3}, i=1,2, \ldots, 6$, such that Eq. (2.12) is satisfied.

3. Monoclinic materials with the symmetry plane at $x_{1}=0$. When the material has a symmetry plane at $x_{1}=0$, Eq. $(2.1)$ reduces to

$$
\left(s_{55}^{\prime} p^{2}+s_{44}^{\prime}\right)\left[s_{11}^{\prime} p^{4}+\left(2 s_{12}^{\prime}+s_{66}^{\prime}\right) p^{2}+s_{22}^{\prime}\right]-\left[\left(s_{14}^{\prime}+s_{56}^{\prime}\right) p^{2}+s_{24}^{\prime}\right]^{2}=0 .
$$

If the eigenvalues $p$ are given by Eq. (1.7), then Eq. (3.1) must have the expression

$$
\left(p^{2}+1\right)^{3}=p^{6}+3 p^{4}+3 p^{2}+1=0 .
$$

Therefore, the coefficient of the $p^{6}$ term in Eq. (3.1) is equal to the constant terms in Eq. (3.1) while the coefficient of the $p^{6}$ term multiplied by 3 is equal to the coefficients of the $p^{2}$ and $p^{4}$ terms. Thus,

$$
\begin{array}{r}
s_{11}^{\prime} s_{55}^{\prime}=s_{22}^{\prime} s_{44}^{\prime}-\left(s_{24}^{\prime}\right)^{2}, \\
3 s_{11}^{\prime} s_{55}^{\prime}=s_{22}^{\prime} s_{55}^{\prime}+s_{44}^{\prime}\left(2 s_{12}^{\prime}+s_{66}^{\prime}\right)-2 s_{24}^{\prime}\left(s_{14}^{\prime}+s_{56}^{\prime}\right), \\
3 s_{11}^{\prime} s_{55}^{\prime}=s_{11}^{\prime} s_{44}^{\prime}+s_{55}^{\prime}\left(2 s_{12}^{\prime}+s_{66}^{\prime}\right)-\left(s_{14}^{\prime}+s_{56}^{\prime}\right)^{2} .
\end{array}
$$


Equation (3.3) gives

$$
\left(s_{24}^{\prime}\right)^{2}=s_{22}^{\prime} s_{44}^{\prime}-s_{11}^{\prime} s_{55}^{\prime} .
$$

Elimination of the term $\left(2 s_{12}^{\prime}+s_{66}^{\prime}\right)$ between Eqs. (3.4) and (3.5) leads to a quadratic equation for $\left(s_{14}^{\prime}+s_{56}^{\prime}\right)$. Making use of Eq. (3.6) it can be shown that

$$
s_{44}^{\prime}\left(s_{14}^{\prime}+s_{56}^{\prime}\right)=s_{24}^{\prime} s_{55}^{\prime} \pm \sqrt{s_{11}^{\prime}\left(s_{44}^{\prime}-s_{55}^{\prime}\right)^{3}} .
$$

Finally, inserting Eq. (3.7) in Eq. (3.4) and using Eq. (3.6) yields

$$
\begin{aligned}
\left(s_{44}^{\prime}\right)^{2}\left(2 s_{12}^{\prime}+s_{66}^{\prime}\right)= & s_{55}^{\prime}\left(s_{22}^{\prime} s_{44}^{\prime}+3 s_{11}^{\prime} s_{44}^{\prime}-2 s_{11}^{\prime} s_{55}^{\prime}\right) \\
& \pm 2 s_{24}^{\prime} \sqrt{s_{11}^{\prime}\left(s_{44}^{\prime}-s_{55}^{\prime}\right)^{3}} .
\end{aligned}
$$

The result is that we may prescribe all components of $s_{i j}^{\prime}$ except $s_{24}^{\prime}, s_{14}^{\prime}$, and $s_{12}^{\prime}$ (or $s_{66}^{\prime}$ ) which are determined from Eqs. (3.6), (3.7), and (3.8). If $s_{i j}^{\prime}$ so obtained is positive definite, we use the procedure outlined at the end of the previous section to determine a complete $C_{i j}$ or $s_{i j}$ which is positive definite. We thus have an anisotropic elastic material whose eigenvalues are given by Eq. (1.7).

To show that it is possible to find a positive definite $s_{i j}^{\prime}$, consider the special case

$$
s_{44}^{\prime}=s_{55}^{\prime} .
$$

Equations (3.6)-(3.8) are simplified to

$$
\begin{gathered}
\left(s_{24}^{\prime}\right)^{2}=\left(s_{22}^{\prime}-s_{11}^{\prime}\right) s_{55}^{\prime}, \\
s_{14}^{\prime}+s_{56}^{\prime}=s_{24}^{\prime}, \\
2 s_{12}^{\prime}+s_{66}^{\prime}=s_{22}^{\prime}+s_{11}^{\prime} .
\end{gathered}
$$

Let

$$
s_{22}^{\prime}=\alpha s_{11}^{\prime}, \quad s_{11}^{\prime}=\gamma s_{55}^{\prime}, \quad s_{56}^{\prime}=s_{12}^{\prime}=0,
$$

where $\alpha$ and $\gamma$ are arbitrary positive constants. We then have

$$
\mathbf{s}^{\prime}=s_{55}^{\prime}\left[\begin{array}{ccccc}
\gamma & 0 & \psi & 0 & 0 \\
0 & \alpha \gamma & \psi & 0 & 0 \\
\psi & \psi & 1 & 0 & 0 \\
0 & 0 & 0 & 1 & 0 \\
0 & 0 & 0 & 0 & \gamma(\alpha+1)
\end{array}\right],
$$

where

$$
\psi= \pm \sqrt{\gamma(\alpha-1)}, \quad \alpha \geq 1 .
$$

The matrix $\mathbf{s}^{\prime}$ is positive definite if the determinants of the five leading principal minors are positive [6]. If $s_{55}^{\prime}, \gamma$, and $\alpha$ are positive, the only remaining condition is

$$
\Delta=\left|\begin{array}{ccc}
\gamma & 0 & \psi \\
0 & \alpha \gamma & \psi \\
\psi & \psi & 1
\end{array}\right|>0 .
$$


It can be shown that

$$
\Delta=\gamma^{2}\left(1+\alpha-\alpha^{2}\right)=\gamma^{2}\left[\frac{\sqrt{5}-1}{2}+\alpha\right]\left[\frac{\sqrt{5}+1}{2}-\alpha\right] \text {. }
$$

In conjunction with Eq. (3.15) $)_{2}$, the $\mathbf{s}^{\prime}$ of Eq. (3.14) is positive definite if

$$
s_{55}^{\prime}>0, \quad \gamma>0, \quad \frac{\sqrt{5}+1}{2}>\alpha \geq 1 .
$$

Therefore, there are infinitely many anisotropic materials for which the eigenvalues are given by Eq. (1.7).

The inverse of $\mathbf{s}^{\prime}$ can be shown to be

$$
\mathbf{C}^{0}=\frac{1}{s_{55}^{\prime} \Delta}\left[\begin{array}{ccccc}
\gamma & \psi^{2} & -\alpha \gamma \psi & 0 & 0 \\
\psi^{2} & \gamma-\psi^{2} & -\gamma \psi & 0 & 0 \\
-\alpha \gamma \psi & -\gamma \psi & \alpha \gamma^{2} & 0 & 0 \\
0 & 0 & 0 & \Delta & 0 \\
0 & 0 & 0 & 0 & \frac{\Delta}{\gamma(\alpha+1)}
\end{array}\right]
$$

and Eq. (1.4) for $p=i$ becomes

$$
\left[\begin{array}{ccc}
\alpha^{2} & i \alpha & -i \alpha(\alpha+1) \psi \\
i \alpha & -1 & (\alpha+1) \psi \\
-i \alpha(\alpha+1) \psi & (\alpha+1) \psi & -(\alpha+1)^{2} \psi^{2}
\end{array}\right] \mathbf{a}=\mathbf{0}
$$

It is seen that the three columns of the $3 \times 3$ matrix on the left are all proportional to each other. Therefore, there are only two independent eigenvectors a for the triple eigenvalue $p=i$. The system is nonsemisimple [7,8]. The general solution for a is

$$
\mathbf{a}^{\mathrm{T}}=\left(k_{1}, i \alpha k_{1}+(\alpha+1) \psi k_{2}, k_{2}\right)
$$

where $k_{1}$ and $k_{2}$ are arbitrary constants.

A more general matrix $s^{\prime}$ can be obtained by considering nonzero $s_{12}^{\prime}$ and $s_{56}^{\prime}$. Instead of Eqs. $(3.13)_{3,4}$ let

$$
s_{12}^{\prime}=\nu s_{55}^{\prime}, \quad s_{56}^{\prime}=\varepsilon s_{55}^{\prime},
$$

where $\nu$ and $\varepsilon$ are constants. We then have

$$
\mathbf{s}^{\prime}=s_{55}^{\prime}\left[\begin{array}{ccccc}
\gamma & \nu & \psi-\varepsilon & 0 & 0 \\
\nu & \alpha \gamma & \psi & 0 & 0 \\
\psi-\varepsilon & \psi & 1 & 0 & 0 \\
0 & 0 & 0 & 1 & \varepsilon \\
0 & 0 & 0 & \varepsilon & \phi
\end{array}\right]
$$

where

$$
\phi=\gamma(\alpha+1)-2 \nu
$$

The matrix $\mathbf{s}^{\prime}$ of Eq. (3.21) is positive definite if, in addition to Eqs. $(3.15)_{2}$ and $(3.17)_{1,2}$,

$$
\begin{gathered}
\phi-\varepsilon^{2}=\gamma(\alpha+1)-2 \nu-\varepsilon^{2}>0, \\
\alpha \gamma^{2}-\nu^{2}>0, \quad \Delta^{\prime}>0,
\end{gathered}
$$


where

$$
\Delta^{\prime}=\left|\begin{array}{ccc}
\gamma & \nu & \psi-\varepsilon \\
\nu & \alpha \gamma & \psi \\
\psi-\varepsilon & \psi & 1
\end{array}\right| .
$$

It can be shown that

$$
\Delta^{\prime}=\frac{1}{\alpha}\left(\alpha \gamma^{2}-\nu^{2}\right)-\frac{1}{\alpha \gamma}[\psi \nu+\alpha \gamma(\varepsilon-\psi)]^{2} .
$$

Therefore Eqs. $(3.22)_{1,3}$ are equivalent to

$$
\begin{gathered}
\gamma(\alpha+1)>2 \nu+\varepsilon^{2}, \\
\alpha \gamma^{2}-\nu^{2}>\frac{1}{\gamma}[\psi \nu+\alpha \gamma(\varepsilon-\psi)]^{2} .
\end{gathered}
$$

It is seen that Eq. (3.25) implies Eq. (3.22) $)_{2}$. We will show in Sec. 7 that Eq. (3.25) also implies Eq. (3.24). Therefore, the conditions for the $\mathbf{s}^{\prime}$ of Eq. (3.21) to be positive definite are Eqs. $(3.15)_{2},(3.17)_{1,2}$, and (3.25). In the special case in which $\nu=\varepsilon=0$, Eqs. $(3.15)_{2}$ and (3.25) reduce to $(3.17)_{3}$. The inverse of $\mathbf{s}^{\prime}$ in Eq. (3.21) is given in Eq. (6.4) with $\beta=1$; and it is shown there that the associated eigenrelation is nonsemisimple.

The condition (3.25) has a simple geometrical interpretation. We show in Sec. 7 that $(\varepsilon, \nu)$ satisfies Eq. (3.25) if it is inside the region bounded by an ellipse; see Fig. 1 .

4. Monoclinic materials with the symmetry plane at $x_{2}=0$. Monoclinic materials with the symmetry plane at $x_{2}=0$ can be obtained from monoclinic materials with the symmetry plane at $x_{1}=0$ by rotating the coordinate system about the $x_{3}$ axis by an angle $\theta=\pi / 2$. It can be shown [9] that, if $p= \pm i$ are the eigenvalues before the coordinate transformation, $p= \pm i$ are the eigenvalues after the coordinate transformation. In fact, $p= \pm i$ for any $\theta$, i.e., for any coordinate transformation which is a rotation about the $x_{3}$-axis. Therefore, $p= \pm i$ can be the triple eigenvalues for monoclinic materials whose plane of symmetry is any plane that contains the $x_{3}$ axis. We hasten to add that, while $p= \pm i$ are invariant with $\theta$, the associated eigenvectors a in general are not. As a result, the Barnett-Lothe tensors [7] are in general not invariant with $\theta[10]$ when $p= \pm i$.

5. Monoclinic materials with the symmetry plane at $x_{3}=0$. For monoclinic materials with the symmetry plane at $x_{3}=0$, Eq. (2.1) reduces to a quadratic equation

$$
s_{55}^{\prime} p^{2}-2 s_{45}^{\prime} p+s_{44}^{\prime}=0
$$

and a quartic equation

$$
s_{11}^{\prime} p^{4}-2 s_{16}^{\prime} p^{3}+\left(2 s_{12}^{\prime}+s_{66}^{\prime}\right) p^{2}-2 s_{26}^{\prime} p+s_{22}^{\prime}=0 .
$$

It is readily shown that if $p= \pm i$ are the only roots of Eqs. (5.1) and (5.2), then

$$
\begin{gathered}
s_{45}^{\prime}=s_{16}^{\prime}=s_{26}^{\prime}=0, \quad s_{44}^{\prime}=s_{55}^{\prime}, \\
s_{11}^{\prime}=s_{22}^{\prime}, \quad s_{66}^{\prime}=2\left(s_{11}^{\prime}-s_{12}^{\prime}\right) .
\end{gathered}
$$


These are the conditions for transversely isotropic materials with the axis of symmetry at the $x_{3}$-axis. Therefore, transversely isotropic materials with the axis of symmetry at the $x_{3}$-axis are the only anisotropic materials with the plane of symmetry at $x_{3}=0$ for which $p= \pm i$ are the triple eigenvalues. Of course, $s_{i j}$ determined from Eq. (2.3) can still represent anisotropic materials other than transversely isotropic materials if $s_{i 3}, i=1,2, \ldots, 6$, are chosen arbitrarily. However, as we stated in Sec. 2, we exclude these trivial cases.

6. Anisotropic materials with an arbitrary $p$ of multiplicity three. In this section we investigate anisotropic materials that possess any three identical pairs of eigenvalues. It suffices to consider

$$
p_{1}=p_{2}=p_{3}=i \beta,
$$

where $\beta$ is a positive constant. This is so because, when $p$ is given by Eq. (6.1), $p(\theta)$ in a rotated coordinate system is [7-9]

$$
p(\theta)=\frac{i \beta \cos \theta-\sin \theta}{i \beta \sin \theta+\cos \theta}=\frac{\left(\beta^{2}-1\right) \sin \theta \cos \theta+i \beta}{\beta^{2} \sin ^{2} \theta+\cos ^{2} \theta} .
$$

With $\beta$ and $\theta$ at our disposal, $p(\theta)$ can be chosen arbitrarily. If $p=i \beta$ is an eigenvalue of multiplicity three, $p(\theta)$ is also an eigenvalue of multiplicity three [9]; in particular, if $\beta=1$, then $p(\theta)=i$ for all $\theta$.

For monoclinic materials with the symmetry plane at $x_{1}=0$, the analyses presented in Sec. 3 can be repeated with Eq. (3.2) replaced by

$$
\left(p^{2}+\beta^{2}\right)^{3}=p^{6}+3 \beta^{2} p^{4}+3 \beta^{4} p^{2}+\beta^{6}=0 .
$$

Equations (3.3)-(3.13) remain valid if we replace $s_{11}^{\prime}, s_{22}^{\prime}, s_{24}^{\prime}$, and $s_{44}^{\prime}$ by

$$
\beta^{2} s_{11}^{\prime}, \beta^{-2} s_{22}^{\prime}, \beta^{-2} s_{24}^{\prime} \text {, and } \beta^{-2} s_{44}^{\prime},
$$

respectively. The rest of the equations in Sec. 3, except Eq. (3.14) and Eqs. (3.18)(3.21), also remain valid. Since Eqs. (3.18)-(3.20) are related to the $\mathbf{s}^{\prime}$ in Eq. (3.14) which is a special case of the $\mathbf{s}^{\prime}$ in Eq. (3.21), we will discuss the modifications of Eq. (3.21).

When the eigenvalues are given by Eq. (6.1), Eq. (3.21) is replaced by

$$
\mathbf{s}^{\prime}=s_{55}^{\prime}\left[\begin{array}{ccccc}
\gamma \beta^{-2} & \nu & \psi-\varepsilon & 0 & 0 \\
\nu & \alpha \gamma \beta^{2} & \psi \beta^{2} & 0 & 0 \\
\psi-\varepsilon & \psi \beta^{2} & \beta^{2} & 0 & 0 \\
0 & 0 & 0 & 1 & \varepsilon \\
0 & 0 & 0 & \varepsilon & \phi
\end{array}\right] .
$$

The conditions for the above matrix to be positive definite are identical to those for the matrix $\mathbf{s}^{\prime}$ of Eq. (3.21), namely, Eqs. $(3.15)_{2},(3.17)_{1,2}$, and (3.25). The inverse 
of $\mathbf{s}^{\prime}$ in Eq. (6.3) is

$$
\mathbf{C}^{0}=\frac{1}{s_{55}^{\prime} \Delta^{\prime}}\left[\begin{array}{ccccc}
\gamma \beta^{2} & \psi \xi-\nu & \nu \psi-\alpha \gamma \xi & 0 & 0 \\
\psi \xi-\nu & \left(\gamma-\xi^{2}\right) \beta^{-2} & (\nu \xi-\gamma \psi) \beta^{-2} & 0 & 0 \\
\nu \psi-\alpha \gamma \xi & (\nu \xi-\gamma \psi) \beta^{-2} & \left(\alpha \gamma^{2}-\nu^{2}\right) \beta^{-2} & 0 & 0 \\
0 & 0 & 0 & \phi \Delta^{\prime} \eta^{-1} & -\varepsilon \Delta^{\prime} \eta^{-1} \\
0 & 0 & 0 & -\varepsilon \Delta^{\prime} \eta^{-1} & \Delta^{\prime} \eta^{-1}
\end{array}\right]
$$

where $\Delta^{\prime}$ is given by Eqs. (3.23) and $\xi=\psi-\varepsilon, \eta=\phi-\varepsilon^{2}$. The eigenrelation (1.4) for $p=i \beta$ for the $\mathbf{C}^{0}$ given by Eq. (6.4) can be shown to have the expression

$$
\left[\begin{array}{ccc}
\beta^{2} X^{2} & i \beta X Y & -i \beta X Z \\
i \beta X Y & -Y^{2} & Y Z \\
-i \beta X Z & Y Z & -Z^{2}
\end{array}\right] \mathbf{a}=\mathbf{0},
$$

where

$$
X=\gamma \alpha-\nu-\varepsilon \psi, \quad Y=\gamma-\nu+\varepsilon(\psi-\varepsilon), \quad Z=\psi \phi-\varepsilon(\gamma \alpha-\nu) .
$$

It can be verified that, subject to the conditions $(3.15)_{2},(3.17)_{1,2}$, and (3.25), $X, Y$, and $Z$ cannot all vanish. Therefore, there are only two independent eigenvectors for the triple eigenvalues $p=i \beta$, and the eigenrelation is nonsemisimple.

For monoclinic materials with the symmetry plane at $x_{3}=0$ it is fairly straightforward to show that, if the eigenvalues are given by Eq. (6.1),

$$
\mathbf{s}^{\prime}=s_{55}^{\prime}\left[\begin{array}{ccccc}
\gamma \beta^{-2} & \nu & 0 & 0 & 0 \\
\nu & \gamma \beta^{2} & 0 & 0 & 0 \\
0 & 0 & \beta^{2} & 0 & 0 \\
0 & 0 & 0 & 1 & 0 \\
0 & 0 & 0 & 0 & 2(\gamma-\nu)
\end{array}\right]
$$

and

$$
\mathbf{C}^{0}=\frac{1}{s_{55}^{\prime}\left(\gamma^{2}-\nu^{2}\right)}\left[\begin{array}{ccccc}
\gamma \beta^{2} & -\nu & 0 & 0 & 0 \\
-\nu & \gamma \beta^{-2} & 0 & 0 & 0 \\
0 & 0 & \left(\gamma^{2}-\nu^{2}\right) \beta^{-2} & 0 & 0 \\
0 & 0 & 0 & \left(\gamma^{2}-\nu^{2}\right) & 0 \\
0 & 0 & 0 & 0 & (\gamma+\nu) / 2
\end{array}\right],
$$

where $\gamma$ and $\nu$ are arbitrary constants. For the matrices $\mathbf{s}^{\prime}$ and $\mathbf{C}^{0}$ to be positive definite, $\gamma>|\nu| \geq 0$. Notice that Eqs. (6.6), (6.7) can be deduced from Eqs. (6.3), (6.4) by setting $\varepsilon=0$ and $\alpha=1$. When $\beta=1$, Eqs. (6.6) and (6.7) represent transversely isotropic materials with the axis of symmetry at the $x_{3}$-axis. This confirms the result obtained in Sec. 5.

7. Discussions. Anisotropic elastic materials represented by the $\mathbf{s}^{\prime}$ of Eq. (6.3) or the $\mathbf{C}^{0}$ of Eq. (6.4) encompass all other $\mathbf{s}^{\prime}$ and $\mathbf{C}^{0}$ considered in this paper. The conditions for the $\mathbf{s}^{\prime}$ of Eq. (6.3) to be positive definite are identical to those for the $\mathbf{s}^{\prime}$ of Eq. (3.21). They are given by

$$
s_{55}^{\prime}>0, \quad \gamma>0, \quad \alpha \geq 1
$$




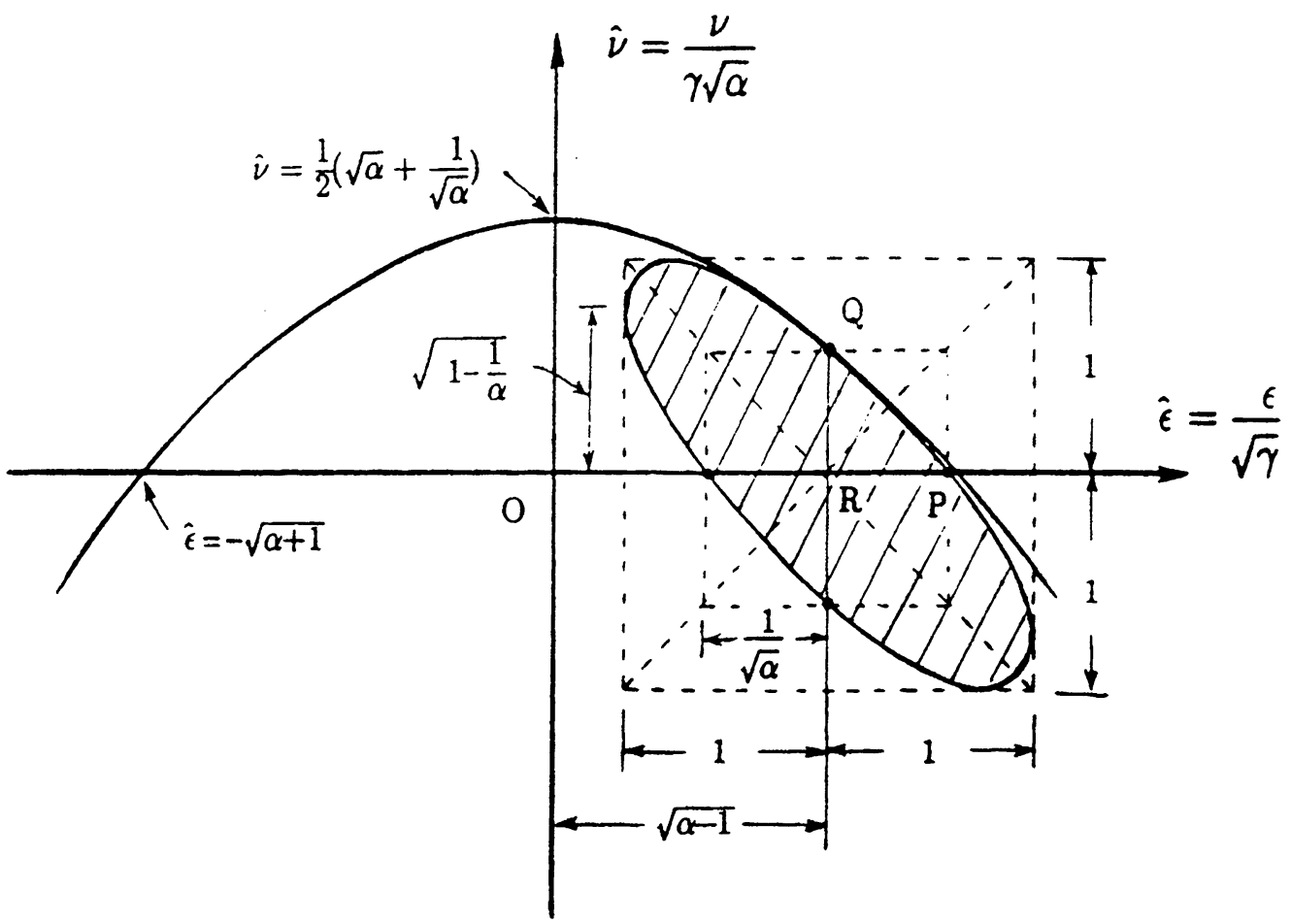

FIG. 1. The region inside the ellipse satisfies the condition (7.4) for the matrix $\mathbf{s}^{\prime}$ of Eq. (6.3) to be positive definite. (Drawn for $\alpha>2$ and $\hat{\psi}>0$.)

and Eqs. (3.24) and (3.25). Conditions (3.24) and (3.25) involve four parameters $\alpha, \gamma, \varepsilon$, and $\nu$. They can be reduced to three parameters $\alpha, \hat{\varepsilon}$, and $\hat{\nu}$ where

$$
\hat{\varepsilon}=\frac{\varepsilon}{\sqrt{\gamma}}, \quad \hat{\nu}=\frac{\nu}{\gamma \sqrt{\alpha}} .
$$

Conditions (3.24) and (3.25) become

where

$$
\begin{gathered}
\sqrt{\alpha} \hat{\nu}<\frac{1}{2}\left(\alpha+1-\hat{\varepsilon}^{2}\right), \\
{[\hat{\psi} \hat{\nu}+\sqrt{\alpha}(\hat{\varepsilon}-\hat{\psi})]^{2}<1-\hat{\nu}^{2},}
\end{gathered}
$$

$$
\hat{\psi}= \pm \sqrt{\alpha-1} \text {. }
$$

For the purpose of the following discussions, it is more transparent to write Eq. (7.4) as

$$
\alpha \hat{\nu}^{2}+2 \sqrt{\alpha} \hat{\psi} \hat{\nu}(\hat{\varepsilon}-\hat{\psi})+\alpha(\hat{\varepsilon}-\hat{\psi})^{2}-1<0 .
$$

When the inequalities are replaced by equalities, Eqs. (7.3) and (7.6) represent, respectively, a parabola and an ellipse in the $(\hat{\varepsilon}, \hat{\nu})$-plane; see Fig. 1. The condition (7.3) implies that $(\hat{\varepsilon}, \hat{\nu})$ lies below the parabola, while Eq. (7.6) tells us that $(\hat{\varepsilon}, \hat{\nu})$ lies inside the ellipse.

The geometry of the parabola and the ellipse depend on the parameter $\alpha$. The ellipse has its center $R$ located at $(\hat{\varepsilon}, \hat{\nu})=(\hat{\psi}, 0)$. Figure 1 shows the ellipse for 
$\alpha>2$ and $\hat{\psi}=\sqrt{\alpha-1}$. If $\hat{\psi}=-\sqrt{\alpha-1}$, the center $R$ of the ellipse is located on the negative $\hat{\varepsilon}$-axis and is the mirror image with respect to the $\hat{\nu}$-axis of the ellipse shown in Fig. 1. The ellipse intersects the positive $\hat{\nu}$-axis twice when

$$
\frac{\sqrt{5}+1}{2} \leq \alpha<2
$$

and contains the origin $(\hat{\varepsilon}, \hat{\nu})=(0,0)$ inside the ellipse when

$$
1 \leq \alpha<\frac{\sqrt{5}+1}{2} \text {. }
$$

It intersects the $\hat{\varepsilon}$-axis at $\hat{\varepsilon}=\hat{\psi} \pm \alpha^{-1 / 2}$ and the line $\hat{\varepsilon}=\hat{\psi}$ at $\hat{\nu}= \pm \alpha^{-1 / 2}$. The ellipse is inscribed in a square of sides two units as shown in Fig. 1. This can be verified when we regard Eq. (7.6) as a quadratic equation for $\hat{\nu}$ and show that the two roots of $\hat{\nu}$ coincide at $\hat{\varepsilon}-\hat{\psi}= \pm 1$. Likewise, regarding Eq. (7.6) as a quadratic equation for $\hat{\varepsilon}$, the two roots of $\hat{\varepsilon}$ coincide at $\hat{\nu}= \pm 1$. The principal axes of the ellipse are along the diagonals of the square. The major and the minor axes have the radii

$$
\left[1+\sqrt{1-\frac{1}{\alpha}}\right]^{1 / 2} \text { and }\left[1-\sqrt{1-\frac{1}{\alpha}}\right]^{1 / 2},
$$

respectively. The ellipse becomes a circle of unit radius with its center at the origin when $\alpha=1$ and degenerates into a diagonal line of the square when $\alpha=\infty$.

We now show that Eq. (7.4) implies Eq. (7.3). To this end, we write Eq. (7.6) as

$$
[\sqrt{\alpha} \hat{\nu}+\hat{\psi}(\hat{\varepsilon}-\hat{\psi})]^{2}<1-(\hat{\varepsilon}-\hat{\psi})^{2}
$$

which gives

$$
-\hat{\psi}(\hat{\varepsilon}-\hat{\psi})-\sqrt{1-(\hat{\varepsilon}-\hat{\psi})^{2}}<\sqrt{\alpha} \hat{\nu}<-\hat{\psi}(\hat{\varepsilon}-\hat{\psi})+\sqrt{1-(\hat{\varepsilon}-\hat{\psi})^{2}} .
$$

Making use of Eq. (7.5) and noting that

$$
\sqrt{1-(\hat{\varepsilon}-\hat{\psi})^{2}} \leq 1-\frac{1}{2}(\hat{\varepsilon}-\hat{\psi})^{2},
$$

it can be shown that the right-hand side of the second inequality in Eq. (7.7) is equal to or less than the right-hand side of the inequality in Eq. (7.3). They are equal when $\hat{\varepsilon}=\hat{\psi}$. Therefore, Eq. (7.4) implies Eq. (7.3). This also means that the parabola and the ellipse do not intersect except at $\hat{\varepsilon}=\hat{\psi}$ where they are in contact (see the point $Q$ in Fig. 1).

When the conditions in Eqs. (7.1) are satisfied and the ellipse is drawn, one can choose any $(\hat{\varepsilon}, \hat{\nu})$ that lies inside the ellipse. The matrix $\mathbf{s}^{\prime}$ of Eq. $(6.3)$ is positive definite.

Finally, we point out that one-component surface waves, which exist only in certain anisotropic elastic materials, can propagate in the materials given by Eq. (6.3). The conditions for the existence of one-component surface waves [11-13] for the materials given by Eq. (6.3) can be shown to be

$$
\gamma=\beta^{2}, \quad \nu=0, \quad \varepsilon(\varepsilon-\psi)>0 .
$$


The last two conditions imply that $(\hat{\varepsilon}, \hat{\nu})$ in Fig. 1 is limited to the segment of the $\hat{\varepsilon}$-axis between the points $P$ and $R$. If the ellipse contains the origin, the entire $\hat{\varepsilon}$-axis inside the ellipse except the segment between the origin and the center of the ellipse satisfies the last two conditions of Eqs. (7.8).

Acknowledgments. The work presented here is supported by the U. S. Army Research Office through grant DAAL 03-91-G-0082.

\section{REFERENCES}

[1] J. D. Eshelby, W. T. Read, and W. Shockley, Anisotropic elasticity with applications to dislocation theory, Acta Metall. 1, 251-259 (1953)

[2] A. N. Stroh, Dislocations and cracks in anisotropic elasticity, Philos. Mag. 3, 625-646 (1958)

[3] S. G. Lekhnitskii, Theory of elasticity of an anisotropic body, Mir Pub. Moscow, 1981

[4] T. C. T. Ting, Barnett-Lothe tensors and their associated tensors for monoclinic materials with the symmetry plane at $x_{3}=0, \mathrm{~J}$. Elasticity 27, 143-165 (1992)

[5] S. C. Cowin and M. M. Mehrabadi, On the identification of material symmetry for anisotropic elastic materials, Quart J. Mech. Appl. Math. 40, 451-476 (1987)

[6] F. E. Hohn, Elementary Matrix Algebra, Macmillan, New York, 1964, pp. 337-340

[7] D. M. Barnett and J. Lothe, Synthesis of the sextic and the integral formalism for dislocations, Greens functions, and surface waves in anisotropic elastic solids, Physica Norvegica 7, 13-19 (1973)

[8] P. Chadwick and G. D. Smith, Foundations of the theory of surface waves in anisotropic elastic materials, Adv. Appl. Mech. 17, 303-376 (1977)

[9] T. C. T. Ting, Effects of change of reference coordinates on the stress analysis of anisotropic elastic materials, Internat. J. Solids Structures 18, 139-152 (1982)

[10] T. C. T. Ting, The Stroh formalism and certain invariances in two-dimensional anisotropic elasticity in Modern Theory of Anisotropic Elasticity and Applications (J. J. Wu, T. C. T. Ting, and D. M. Barnett, eds.), SIAM Proc. Ser., SIAM, Philadelphia, 1991, pp. 3-32

[11] D. M. Barnett and P. Chadwick, The existence of one-component surface waves and exceptional subsequent transonic states of type 2, 4 and E1 in anisotropic elastic media in Modern Theory of Anisotropic Elasticity and Applications (J. J. Wu, T. C. T. Ting, and D. M. Barnett, eds.), SIAM Proc. Ser., SIAM, Philadelphia, 1991, pp. 199-214

[12] D. M. Barnett, P. Chadwick, and J. Lothe, The behavior of elastic surface waves polarized in a plane of material symmetry. I. Addendum, Proc. Roy. Soc. London Ser. A 433, 699-710 (1991)

[13] T. C. T. Ting, The motion of one-component surface waves, The P. Chadwick Symposium Volume, J. Mech. Phys. Solids 40, 1637-1650 (1992) 